\title{
Relato de Experiências em Espaços Makers nas Escolas do Ensino Fundamental
}

\author{
Jadson do Prado Rafalski - Prefeitura Municipal de Vila Velha - \\ jadsonrafalski@gmail.com \\ Maria Aparecida de Faria da Silva - Prefeitura Municipal de Vila Velha - \\ cidadfaria@hotmail.com \\ Ramon Rosa Maia Vieira Júnior - Universidade Federal do Rio Grande do Sul \\ - ramonwaia@gmail.com
}

\begin{abstract}
Resumo. A Robótica Educativa surgiu com a proposta de tornar-se lúdicas e interessantes explorando conceitos presentes no currículo escolar. This work is related a youth in an instrumental missionary in ensino fundamental in school schools of Vila Velha in the Espírito Santo. Para o desenvolvimento deste relato de experiências em múltiplos projetos, desafios e trabalhos que resolvem problemas integrando como múltiplas disciplinas.

Palavras-chave: Espaço Maker, Aprendizado, Laboratório, Robótica.

Abstract. Educational Robotics emerged with the proposal of becoming playful and interesting exploring concepts present in the school curriculum. This work is related to youth in an instrumental missionary in elementary school in school schools of Vila Velha in the Espírito Santo. For the development of this report of experiences in multiple projects, challenges and works that solve problems integrating as multiple disciplines
\end{abstract}

Keywords: Space Maker, learning, laboratory, robotics.

\section{Introdução}

Vale a pena começar a dizer "os tempos mudaram". As crianças de hoje estão crescendo no período totalmente digital. Seu desenvolvimento avança com a tecnologia, que lhes permite adaptar-se facilmente a qualquer avanço. É por isso que a educação tem que seguir em frente continuando o progresso, se reinventar a cada dia.

As interfaces digitais e os componentes eletrônicos estão, cada vez mais, permitindo novas formas de experimentar e criar. Enquanto a tecnologia ganha complexidade por meio da microeletrônica, processamento e softwares, as interfaces (físicas e digitais) oferecem progressivamente mais facilidades de uso (usabilidade). Diferentes componentes eletrônicos (microcontroladores, kits programáveis, sensores ...) ganham os espaços escolares com interfaces cada vez mais amigáveis, tornando o uso cada vez mais acessível e suas aplicações cada vez mais diferenciadas.

O avanço da eletrônica e das interfaces permitiram subsidiar com novos equipamentos e configuração dos espaços para estruturar e ampliar ambientes em favor de uma educação direcionada a experimentação, com espaço de criação. Como resposta a estas oportunidades, os Espaços Makers encontram na Robótica Educacional uma 
integração a uma pedagogia mais ativa, que centre no sujeito as principais atividades do ensino-aprendizagem.

O espaço maker baseia-se numa prática construtivista para formar uma abordagem construcionista, introduzida por Jean Piaget e desenvolvida por Seymour Papert (PAPERT, 1994). O principal objetivo do construcionismo é fazer com que os alunos criem seus próprios conhecimentos criando e interagindo com objetos físicos. Tem conexões claras com a alfabetização midiática e com a aprendizagem auto-dirigida.

No Mundo, diversas escolas já introduziram o espaço maker como um ambiente de aprendizagem, criando oportunidade de dar sentido ao que estão aprendendo, buscando soluções que gerem aprendizado relacionado com a vida. Os projetos Fab Learn Labs (FAB LEARN LABS, 2018), FabLab kid (FABLAB KID, 2018), Lite (LITE UNIVALI, 2018) e POALab (POALAB, 2018) são exemplos desta união entre o movimento maker e a educação. Nestes espaços tem por objetivo a interdisciplinaridade da aprendizagem, voltado à invenção e a inovação proporcionando uma oportunidade de exercitar e desenvolver a criatividade e a troca de experiências entre os indivíduos.

Nesse cenário de transformações no modo de pensar as práticas de ensino e aprendizagem abordamos neste artigo um relato de experiência dos alunos do ensino fundamental de 10 (dez) escolas públicas de ensino fundamental no município de Vila Velha no Espírito Santo, Brasil que participam de um projeto de robótica no contra turno. O objetivo deste trabalho foi pensar de forma criativa, refletir de maneira sistêmica e trabalhar de modo colaborativo com os alunos do ensino fundamental, apresentando para a comunidade acadêmicas as possibilidades de utilizar a robótica envolvendo outras ciências, criando um ambiente escolar interdisciplinar.

No Brasil a aprendizagem em espaços maker ainda é pouco explorada (BORGES, K.S., FAGUNDES, L.D., \& MENEZES, 2016) e não possui uma proposta pedagógica formalizada, é uma área ainda pouco explorada pela pesquisa. Neste sentido este trabalho tem como objeto de estudo apresentar um relato de experiência do uso da robótica como ferramenta de aprendizado e transformação educacional promovendo a interdisciplinaridade em espaços makers nas escolas do ensino fundamental. Na seção 2 é apresentado a robótica educacional para o currículo interdisciplinar. A seção 3 discorre sobre a metodologia do projeto de robótica. A seção 4 apresenta uma proposta de trabalho com um laboratório de robótica. A seção 5 apresenta os resultados do projeto. A seção 6 traz as considerações finais deste trabalho, indicando os próximos passos da pesquisa.

\section{A Robótica na Educação para o currículo Interdisciplinar}

A revisão da literatura revela que a Robótica é um campo em crescimento com o potencial de impactar significativamente a natureza da educação em ciência e tecnologia em todos os níveis. A Robótica Educacional surgiu como uma ferramenta única de aprendizado que pode oferecer atividades práticas e divertidas em um ambiente de aprendizado atraente que alimenta o interesse e a curiosidade dos alunos (Eguchi, 2010). No entanto, tem sido observado nos últimos anos que o interesse popular em robótica aumentou espantosamente (Benitti, 2012). Além disso, a disponibilidade de robótica para o ensino fundamental está crescendo rapidamente (Mataric, 2004).

A robótica pode ser abordada de maneira inteligente e diferenciada envolvendo os estudantes. Por meio de uma abordagem curricular temática as áreas curriculares são integradas em torno de um tópico especial para aprendizagem e estudadas principalmente por meio de questionamento e comunicação (Alimisis, 2013), podemos ainda acrescentar 
conforme (Alimisis, 2017) que abordagem baseada em projeto os alunos trabalham em grupos para explorar problemas do mundo real; este é, por exemplo, o caso proposto na metodologia desenvolvida pelo projeto europeu TERECoP, Educação Pedagógica em Métodos Pedagógicos Construtivistas Avançados em Robótica, (www.terecop.eu), outra possibilidade é a participação dos estudantes em competições em desafios nos torneios de robótica que ocorrem, como a Olimpíada Brasileira de Robótica (http://www.obr.org.br), FIRST Lego League (http://www.firstlegoleague.org), a RoboCupJunior (http://rcj.robocup.org) e outras competições mundiais.

Para d'Abreu (1999), ao elaborar-se um ambiente de aprendizagem baseado no uso de dispositivos robóticos, pode-se dar tanto o enfoque técnico-industrial quanto o pedagógico-educacional. Considerando-se que:

\begin{abstract}
a ideia central é propiciar ambientes de aprendizagem baseados em dispositivos robóticos que permitem a construção do conhecimento nas diferentes áreas das ciências. Neste enfoque, a robótica pedagógica pode ser definida como a utilização da robótica industrial num contexto onde as atividades de construção e controle de dispositivos, usando kits de montar ou outros materiais, propicia o manuseio de conceitos de ciências em geral num ambiente de sala de aula, nos diferentes níveis de ensino (D'ABREU, 1999, $p$. 1).
\end{abstract}

As principais teorias por trás da robótica educacional são o construtivismo e o construcionismo. Piaget argumenta que a manipulação de artefatos é uma chave para as crianças construírem seus conhecimentos (Piaget, 1972). Papert acrescentou a ideia de que a construção do conhecimento acontece de maneira especialmente eficaz em um contexto em que o indivíduo está conscientemente envolvido na construção, seja um artesanato ou um artefato tecnológico (Papert, 1980).

O papel dos educadores é oferecer oportunidades para que os estudantes se envolvam em explorações práticas e forneçam ferramentas com a finalidade de construir o conhecimento no ambiente de sala de aula. Nesse contexto os alunos têm a oportunidade de exercer a intuição, a experimentação, condições de formular perguntas e hipóteses, tomar consciência, dialogar, instaurar a interação, transformar, descobrir, inventar e construir conhecimento. Aprender com a robótica educacional fornece aos alunos oportunidades para que eles parem, questionem e pensem profundamente sobre tecnologia. Ao projetar, construir, programar e documentar projetos, os estudantes não apenas aprendem como a tecnologia funciona, mas também aplicam as habilidades e o conhecimento de conteúdo aprendidos na escola de maneira significativa e empolgante.

A Robótica Educacional cria um ambiente de aprendizado no qual os estudantes podem interagir com seu ambiente e trabalhar com problemas do mundo real; Nesse sentido, a Robótica Educacional pode ser uma ótima ferramenta para que os estudantes tenham experiências de aprendizado. Projeto de robótica é rica em oportunidades para integrar não apenas a área da tecnologia, mas também muitas outras disciplinas, incluindo alfabetização, estudos sociais, dança, música e arte, ao mesmo tempo que oferece aos alunos a oportunidade de encontrar novas maneiras de trabalhar em equipe com a finalidade de promover habilidades de colaboração compartilhando conhecimento das diversas ciências. A robótica educativa é uma ferramenta de aprendizado que aprimora a experiência do aluno por meio do aprendizado prático. A elaboração de projetos de robótica permite que o estudante tenha um ambiente de aprendizado divertido e estimulante devido à sua natureza prática e à integração da tecnologia. Os estudantes se 
envolvem e contribuem com habilidades e conhecimentos necessários para que eles atinjam seus objetivos, a fim de completar os projetos de seu interesse.

Estudos no campo relatam que a robótica tem um impacto potencial na aprendizagem do aluno em diferentes áreas (Física, Matemática, Engenharia, Informática e mais) e no desenvolvimento pessoal incluindo cognitiva, meta-cognitiva e habilidades sociais, tais como: habilidades de pesquisa, pensamento criativo, tomada de decisão, resolução de problemas, comunicação e habilidades de trabalho em equipe, sendo todas habilidades essenciais necessárias no ambiente de trabalho do século XXI (Eguchi, 2010).

\section{Aspectos Metodológicos do Projeto}

Nossa abordagem metodológica tenta levar em conta os diferentes aspectos envolvidos em um processo de ensino-aprendizagem construtivista, dentro de um contexto escolar concreto.

As teorias construtivistas de Jean Piaget argumentam que a aprendizagem humana não é o resultado de uma transmissão de conhecimento, mas um processo ativo de construção do conhecimento em experiências do mundo real e ligadas a pré conhecimento pessoal (Piaget 1972). O desenho de boas experiências de educação-aprendizagem (construtivistas) com robôs tem que ser feito levando em conta os seguintes pontos:

A experiência com atividades laboratoriais permite que os sujeitos possam experimentar suas teorias em práticas em uma lógica de interação (sujeito $\mathrm{x}$ objeto). $\mathrm{O}$ laboratório de experimentação munido de variados instrumentos facilita as práticas experimentais, promovendo, assim, a criação progressiva de testes. As diferentes tentativas praticadas pelos alunos constituem um ambiente enriquecido de oportunidades de assimilação. Os sucessos e fracasso permitem que as ações iniciais possam ser validadas e/ou reconduzidas a novas ações, isto é a tomada de consciências progressivas, ao ponto de que não somente se alcança um saber-fazer, mas que permita um compreender as razões do seu próprio fazer.

A Pirâmide da Aprendizagem, primeiramente chamada de cone da experiência, traz a percepção de Edgar Dale (1946), realizou diversos estudos nos quais verificou que o conhecimento adquirido ou aprendido após $24 \mathrm{~h}$ para um aluno é de $5 \%$ para palestras, $50 \%$ para discussões em grupo, $75 \%$ para experiências práticas e $90 \%$ para o ensino a pares. Percebe-se que quanto mais ativo o estudante for em suas práticas acadêmicas, maiores são as possibilidades de aprendizagem real e significação social (LALLEY; MILLER, 2007, p.68).

Uma das primeiras atividades propostas no projeto é envolver o estudante por meio de debates e roda de conversa sobre o desenvolvimento de tecnologias e valores sociais. Em um segundo momento, a mesma atividade é focada em atividades práticas, a fim de aumentar a curiosidade, fantasia e lógica nos estudantes. O objetivo é aprender o processo tecnológico como um aspecto positivo utilizando a colaboração entre os sujeitos, dando contribuição própria ao grupo.

Uma segunda atividade é destinada ao planejamento e construção de assuntos de lógica e programação. O projeto tem como perspectiva trazer os conceitos básicos da Ciência da Computação, principalmente da programação, para o ambiente escolar. Os estudantes são convidados para participar de competições e torneios de programação aplicados a robótica. 


\section{O Projeto de Robótica}

Vale ressaltar que não há a disciplina de introdução a robótica no currículo escolar, conforme o sistema de Ensino Brasileiro de Educação (BORGES, K.S., FAGUNDES, L.D., \& MENEZES, 2016), no entanto Papert apresenta em seus estudos a proposta da robótica educacional como uma nova disciplina com o viés construcionista:

O esboço desta nova disciplina surgirá gradualmente, e o problema de situá-la no contexto da Escola e no ambiente de aprendizagem maior, será melhor apresentado quando a tivermos na nossa frente. Apresento aqui uma definição preliminar da disciplina - porém apenas como uma semente para discussão - como aquele grão de conhecimento necessário para que uma criança invente (e, evidentemente, construa) entidades com qualidades evocativamente semelhantes à vida dos mísseis inteligentes. Se este grão constituísse a disciplina inteira um nome adequado seria "engenharia de controle" ou até mesmo "robótica" (Papert, 1994, p.160).

No entanto, o uso da robótica na educação era inviabilizado pela falta de acesso a robôs, ou aos componentes que são necessários para a sua construção devido principalmente, ao alto custo dos materiais e a falta de equipamentos de robótica com facilidade para programar. Com o avanço de iniciativas privadas e livres surgiu alguns componentes utilizados para a construção e manipulação de robôs, alguns dos quais proprietários, com o LEGO Mindstorms EV3 (Mindstorms, 2019), e outros baseados em hardware livre, como o Arduino (Arduino, 2019). Esforços têm mostrado que as escolas nas últimas décadas têm se destacado e os estudantes estão entusiasticamente envolvidos em projetos maker de robótica, atingindo metas de aprendizado e desenvolvimento de habilidades (OLIVEIRA e ARAUJO, 2016).

O principal objetivo deste projeto é relatar a experiência da introdução da Robótica na escola de ensino fundamental $\mathrm{II}^{1}$ como um assunto normal ao currículo de atividades diversificadas, além de ser proposto como uma atividade extra a ser realizada fora do horário escolar.

Os encontros acontecem durante o contra turno, os estudantes não são obrigados a estarem nas aulas uma vez que não existe "chamada" para nota e/ou falta, mas são realizadas propostas de projetos para que os estudantes possam apresentar durante os encontros. Essa maneira de trabalhar está diretamente relacionada a pedagogia de projetos e a autonomia de cada estudante em elaborar o seu "invento".

O objetivo do Projeto de Robótica nas escolas de ensino fundamental (8 a 12 anos de idade) é servir um espaço interdisciplinar de aprendizagem para que os estudantes sejam inventores e criadores. Com a robótica, os estudantes têm uma oportunidade diferente para desenvolver sua capacidade lógica e sua criatividade utilizando raciocínio e do pensamento crítico.

\subsection{Metodologia}

A metodologia adotada durante os encontros seguiu o modelo participativo-colaborativoconstrutivista (Abud, 2003). O modelo participativo possibilita o envolvimento das pessoas no processo de tomada de decisões. O modelo colaborativo possibilita a troca de 
experiência entre dois ou mais sujeitos proporcionando a construção de conhecimentos por meio de discussões, reflexões e tomadas de decisões em conjunto.

Os professores do projeto são servidores da rede municipal de ensino, dos 22 professores 20 são formados em licenciatura e 2 professores em ciências da computação conforme apresentado na tabela 1. Realizamos os encontros de maneira presencial na quarta-feira com duração de 4 horas mensalmente, para realizar uma formação com estes professores nas diversas tecnologias (lógica, programação, eletrônica, projetos, currículo, etc). Nos encontros os professores têm a oportunidade de trocar experiências com outros professores de outras escolas que também participa do projeto de robótica. O ponto negativo é que os professores dividem carga horária com a disciplina chamada tecnologia educacional que faz parte do currículo, e isso comprometia uma formação quinzenal, uma vez que esse professor tinha outras formações para participar. Por fazer parte do currículo essa disciplina os professores tinham que preencher pautas o que comprometia algumas quartas-feiras.

1 - Estudantes a partir dos 11 anos de idade até os 15 anos de idade.

\begin{tabular}{|c|c|}
\hline Formação Acadêmica & Quantidade de Professores \\
\hline Licenciatura em Matemática & 3 \\
\hline $\begin{array}{c}\text { Licenciatura em Língua } \\
\text { Portuguesa }\end{array}$ & 1 \\
\hline Licenciatura em Biologia & 1 \\
\hline Licenciatura em Ed. Física & 1 \\
\hline Pedagogia & 14 \\
\hline Cientista da Computação & 2 \\
\hline
\end{tabular}

Tabela 1 - Quantidade e Formação Acadêmica dos Professores de Robótica

Trabalhar com crianças e pré-adolescentes é necessário usar de recursos lúdicos que sejam atraentes para os mesmos. Para o desenvolvimento de lógica utilizamos os aplicativos Scratch e o Code (Code, 2019), os professores desenvolveram trabalhos durante um mês a dois meses nessas plataformas.

Passando os diversos desafios que eram propostos, os estudantes avançaram para os conceitos de algoritmos descritivos por meio de uma sequência de passos para solucionar problemas, como é feito no fluxograma. Tivemos algumas dificuldades dos estudantes entender alguns conceitos de condicional no fluxograma, quando isso acontecia era necessário uma intervenção da ferramenta Scratch para compreender melhor o conceito.

Após as etapas de desenvolvimento de raciocínio lógico e noções básicas de algoritmos, era apresentado aos estudantes o Arduino, variados sensores eletrônicos e analógicos e o software S4A (S4A, 2019). No decorrer dos encontros, muitas propostas foram desenvolvidas pelos alunos. Sempre era realizado o desafio durante as aulas no final de cada encontro é apresentado pelos estudantes.

Por exemplo, durante um encontro os estudantes tiveram a oportunidade de conhecer o sensor ultrassônico, depois de entender sua aplicação, os estudantes fizeram uma proposta de contextualização sobre o uso deste sensor no dia a dia, então fizeram 
uma proposta de um semáforo que ao ver uma pessoa informa a quantidade de minutos que resta para abrir ou fechar por meio de um display.

Após diversas experimentações, os alunos são desafiados a contextualizar os diversos sensores e o Arduino ao contexto do seu dia a dia, solucionando algum problema. Para isso foram disponibilizados para os alunos das 10 (dez) escolas participantes do projeto de robótica os kits de arduino e materiais de papelaria e artesanato. Os materiais que não tivesse na escola os alunos conseguiram na comunidade local por meio de doações como por exemplo canos do tipo tubo de $100 \mathrm{~mm}$.

Os projetos aconteceram da seguinte forma: os estudantes foram questionados sobre a possibilidade de descrever um problema que poderia ser resolvido utilizando os materiais que foram entregues. Os estudantes já tinham conhecimento prévio dos sensores, arduino, placas, material de artesanato e papelaria. Durante uma semana os estudantes tiveram a oportunidade de pensar em um problema da sua comunidade local ou da sua escola. Sendo assim cada uma escola apresentou uma proposta de problema:

- Escola X o grupo dos estudantes da robótica verificaram que nas aulas de música não havia alguns equipamentos como por exemplo o teclado musical.

- Escola $\mathrm{Z}$ o grupo dos estudantes da robótica analisou que faltava um semáforo próximo a escola, e que este teria que ter um temporizador e um sinal sonoro para facilitar que os alunos deficientes de baixa visão.

- A Escola Y o grupo de estudantes participou de palestra sobre meio ambiente e reciclagem na escola. Assim os alunos despertaram a ideia de unir a robótica com equipamentos inservíveis de tecnologia.

- A Escola W tem um horta escolar porém com dificuldades para realizar o cultivo. O grupo dos alunos esta escola teve ficaram instigados em resolver o problema do cultivo por meio de uma hidroponia.

Durante dois a três meses nas aulas de robótica os estudantes trabalharam em suas escolas para o desenvolvimento do projeto propondo uma solução prototipada para o problema apresentado. Logo em seguida os estudantes realizaram a apresentação de seus protótipos na I Mostra de Robótica das escolas municipais de ensino fundamental. Foram as soluções para os problemas apresentados:

- Para a escola X os estudantes desenvolveram um teclado musical por meio de um teclado musical de banana.

- Para a escola $Z$ os estudantes criaram uma maquete da escola com a avenida e colocaram um semáforo com LED e um sinalizador com o buzzer.

- Na Escola Y os estudantes aproveitaram o material de sucata e transformou em material de decoração. Conforme apresentado na Figura 3, um porta-retratos foi produzido.

- Na Escola W os alunos construíram um protótipo de hidroponia para a horta escolar. 


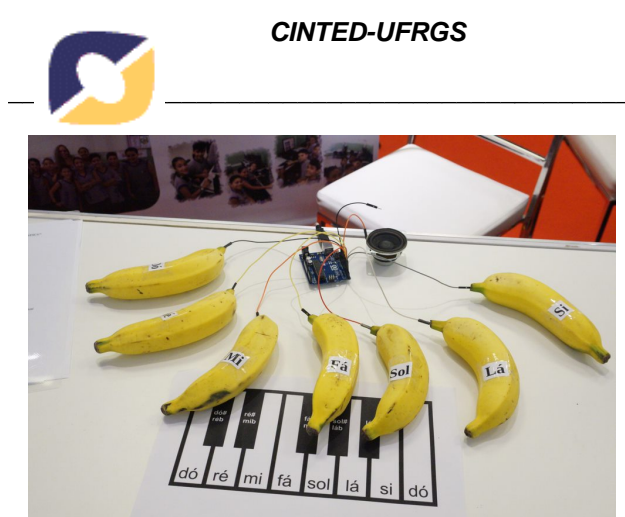

Figura 1 - Criação dos Alunos da Escola $\mathbf{X}$

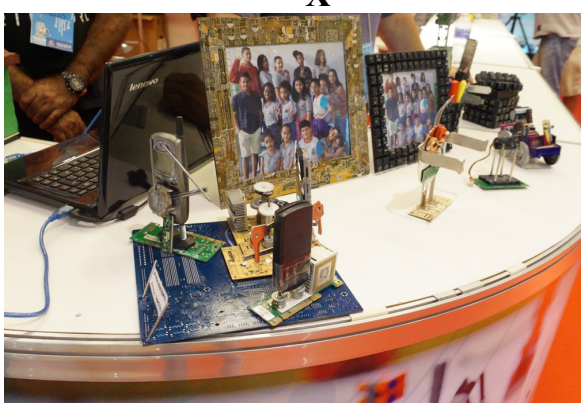

Figura 3 - Criação dos Alunos da Escola Y

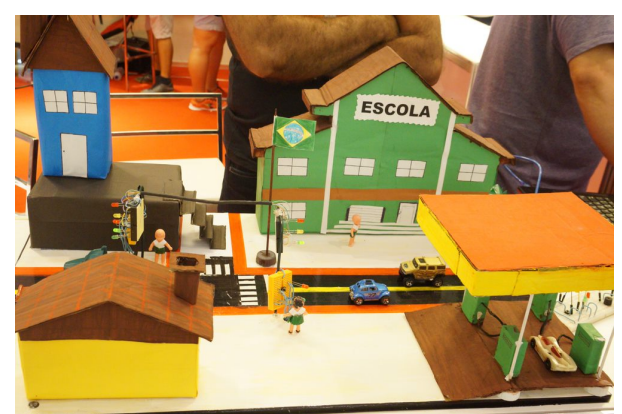

Figura 2 - Criação dos Alunos da Escola Z

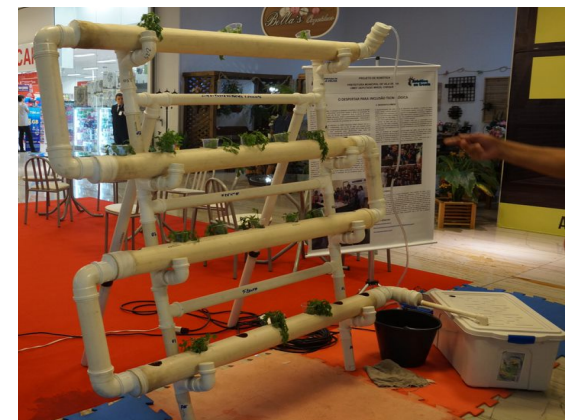

Figura 4 - Criação dos Alunos da Escola W

\section{Resultados do Projeto}

Este artigo apresentou o relato de experiências de encontros no projeto de robótica realizadas com os estudantes da rede municipal de Vila Velha/ES. Por meio dos encontros os estudantes criaram projetos de automação, eletrônica, robótica, etc, criando soluções e prototipando ideias. Os professores e alunos não tinham inicialmente conhecimentos com linguagem/lógica de programação/eletrônica, a busca por esse conhecimento se deu por cada protagonista de acordo com a necessidade do projeto. Ao final da apresentação dos projetos foi aplicado um questionário de satisfação semiestruturado, com perguntas abertas e fechadas, visando coletar informações a respeito dos encontros que aconteceu durante o ano. Participaram do projeto de robótica 16 professores, sendo que destes nenhum destes são formados em áreas exatas.

Os resultados apontaram que o projeto de robótica nas escolas proporcionou, segundo os professores, o desenvolvimento e despertar do estimulo intelectual para os estudantes, melhora o raciocínio lógico-matemático, melhora a capacidade de planejamento e melhora a capacidade de interpretação de textos/compreensão verbal, melhora a interação/socialização, melhora a capacidade de liderança, melhora a motivação. As aulas se tornaram mais prazerosas, uma vez que eram realizadas no contra turno por meio de projetos em que não tinha nota, mas o estudante mostrava interesse em participar e frequentar as aulas. De acordo com os professores, um ponto positivo são as trocas de experiências entre os professores

Duas perguntas realizadas no questionário foram: i. identificar os conteúdos que podem ser trabalhados nos projetos de robótica e os ii. relatos e experiencias dos estudantes que tenha chamado a atenção durante os encontros. Dentre as respostas postadas pelos professores na tabela 1 destaco algumas respostas de 2 professores (as): 


\begin{tabular}{|l|l|}
\hline \multirow{5}{*}{ Professor (a) A } & $\begin{array}{l}\text { Na sua opinião, como os conteúdos da sua disciplina são trabalhados no } \\
\text { Projeto de Robótica? } \\
\text { "Trabalho diretamente com a oficina de robótica e tecnologias } \\
\text { educacionais. Acredito que os alunos que estão frequentando a oficina estão } \\
\text { tendo mais intimidade com computador e desenvolvendo mais habilidades, } \\
\text { tanto na questão de protagonismo, quanto na questão de aprendizagem." }\end{array}$ \\
\cline { 2 - 2 } & $\begin{array}{l}\text { Relate um caso de uma criança que participa(ou) do Projeto de Robótica que } \\
\text { chamou sua atenção positiva ou negativamente. }\end{array}$ \\
& "Tenho um aluno que é bem rebelde na sala de aula convencional. E \\
destaca-se na robótica, e depois de muita conversa ele tem se esforçado pra \\
mudar o comportamento... Acho que a robótica é isso, a gente não precisa \\
cobrar tanto, eles gostam e naturalmente se esforçam para compreender \\
melhor."
\end{tabular}

Tabela 1 - Relato de professores do projeto de robótica

Em geral, os resultados foram positivos, houve uma significativa melhora no comportamento e intelecto dos estudantes durante o encontros do projeto de robótica, ter autonomia, propostas de projetos, utilizar o contra turno das aulas para trabalhar foi um diferencial para a realização efetiva do projeto.

\section{Considerações Finais}

Neste artigo, é feita uma tentativa de introduzir atividades de robótica aos alunos do ensino fundamental, apoiando-os a trabalhar em construções de robóticas simples com Arduino e Lego Mindstorms. Os alunos são convidados a trabalhar em grupos para projetar, construir e programar. Iniciativas como esta apresentada neste artigo vêm seguindo diferentes perspectivas.

Foi visto por meio da análise literária que o movimento maker é capaz de sobrepor a diversas restrições, e pode ser adaptada na sala de aula de maneira que pudesse envolver os estudantes, com a finalidade de promover uma mudança cultural que instigue um espírito criativo e a autoria dos alunos ainda em fase de formação.

\section{Referências}

ABUD, K.M. Um Projeto de Formação Continuada para Professores de História. (Org.) CARVALHO, A.M.P. Formação Continuada de Professores, pp. 86-94, 2003. 
ALIMISIS, D, et al, 2013. Educational robotics: Open questions and new challenges. In Themes in Science \& Technology Education, 6(1), 63-71, 2013.

BORGES, K.S., FAGUNDES, L.D., \& MENEZES, C.S. (2016). "Projetos Maker Como Forma De Estimular O Raciocínio Formal Através Do Pensamento Computacional", Anais do XXII Workshop de Informática na Escola / V Congresso Brasileiro de Informática na Educação.

D'ABREU, J. V. V. Desenvolvimento de Ambientes de Aprendizagem Baseados no Uso de Dispositivos Robóticos. Anais do X Simpósio Brasileiro de Informática na Educação - SBIE99. Curitiba, PR, 1999.

EGUCHI, A. (2010). What is educational robotics? Theories behind it and practical implementation. In D. Gibson \& B. Dodge (eds.), Proceedings of Society for Information Technology \& Teacher Education International Conference 2010 (pp. 4006-4014). Chesapeake, VA: AACE.

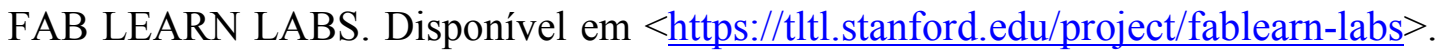
Acesso em 01/06/2018.

FABLAB KID. Disponível em $<$ http://fablabkids.org>. Acesso em 01/06/2018.

LITE U BENITTI, F.B.V., "Exploring the educational poten- tial of robotics in schools: A systematic review," Computers \& Education, vol. 58, no. 3, 2012, 978988. DOI: http://dx.doi.org/10.1016/j.compe- du.2011.10.006.

LALLEY, James; MILLER, Robert H. The Learning Pyramid: Does it point teachers in the right direction? Education, v.128, n.1, p.64-79, 2007.

LITE UNIVALI, Disponível em <http://lite.acad.univali.br/pt/>. Acesso em 01/06/2018.

MATARIC, M.J., "Robotics Education for All Ages," American Association for Artificial Intelligence Spring Symposium on Accessible, Hands-on AI and Robotics Education, 2004.

POALAB, Disponível em <http://www.poalab.net.br $>$. Acesso em 01/06/2018.

PAPERT, S. The Children's Machine: Rethinking School In The Age Of The Computer. New York: BasicBooks, 1994.

PAPERT, S. (1980). Mindstorms: Computers, Children and Powerful Ideas. NY: Basic Books.

PIAGET, J: The Principles of Genetic Epistemology. N.Y.: Basic Books (1972).

PRENSKY, Marc. Disponível em <http://marcprenskyarchive.com/writings/> Acesso em 01 junho de 2018 Digital Natives, Digital Immigrants (texto publicado na sua primeira versão em 2001).

OLIVEIRA, E. J. S., ARAUJO, A. L. S. O., "Pensamento Computacional e Robótica: Um Estudo Sobre Habilidades Desenvolvidas em Oficina de Robótica Educacional", Anais do XXVII Simpósio Brasileiro de Informática na Educação SBIE 2016. 